\title{
A Study of Interpreting Skills from the Perspective of Interpreting Process
}

\author{
Jing Ma \\ Foreign Language Department, Qingdao University of Science and Technology, Qingdao 266001, China

\begin{abstract}
The paper starts from the analysis of the interpreting process, analyzes the problems and factors that may effects the quality of interpreting in different procedures, then focuses on the discussion of four categories of interpreting skills, namely, listening comprehension skills, decoding skills, recording skills, and re-expressing skills to overcome those problems and factors.
\end{abstract}

Index Terms - interpreting skills, interpreting process, comprehension, recording, decoding, re-expressing

\section{INTRODUCTION}

Interpreting as a modern profession has established its status in the international communities. Interpreters help people overcome languages barriers, dispel their suspicion and serve as a bridge in intercultural communication. But, to finish all these tasks, an interpreter should possess special talents and skills and therefore interpreting skills have become one of the hot issues that received wide attention. Also, based on the analysis of interpreting process and all aspects of the teaching of it, the experts, at home and abroad, are generally convinced that it is the prime task and ultimate objective of teaching interpretation to train abilities and skills. Liu Miqing (1999) states that the sooner one gets aware of the significance of the interpretative skills training, the sooner he/she will be good at interpretation.

Yet research on interpretation in our country is still at the primary stage, and no systematic theories have been established to show interpreters and those want-to-be interpreters what kind of skills they must possess and how to possess necessary skills effectively. In this case, the current thesis will probe into the issue of interpreting skills from the perspective of interpreting process.

\section{ANALYSIS OF INTERPRETING PROCESS}

\section{A. The Perception Procedure}

There are mainly two ways for the interpreter to perceive the message: one is auditory perception and the other is visual perception. In this procedure, factors that will influence the quality of the interpretation include the interpreter's listening ability, and the setting.

Compared with the general listening, listening in interpreting is a more complicated and difficult process. The setting, temporal and physical conditions, in which communication takes place also play an important role in the interpretation process. Time constraints for speakers at conferences often lead them to deliver their message at a furious pace. Noisy listening conditions make the reception of the communicating message difficult both for intended receptors and for the interpreter.

\section{B. The Decoding Procedure}

The decoding procedure follows in an almost indiscernible instance, during which the interpreter processes the information stored in the "perceptual auditory storage" and extracts necessary information from both linguistic and non-linguistic codes. It is where the message is born.

In this procedure, factors that will influence the quality of the interpretation include the source, particularly the speaker, the interpreter's linguistic competence and his background knowledge, which includes the knowledge of the world and knowledge of the subject under discussion.

\section{The Recording Procedure}

In this procedure, the interpreter's memory plays a crucial role, and is a decisive factor in whether this procedure is successful or not or at least in how much the interpreter can get from the source's speech. A supplementary way of securing this procedure is note-taking, which seems to be more important in consecutive interpretation.

Recording must be based on comprehension, and it is two folded, mental and written. According to Bao Gang (1998), there are three kinds of memories in human being's information processing system, known as sensory store (perception), short term store (working memory) and long term store (permanent memory) (p.146). Among these three kinds of memories, working memory plays an important part in our work especially in interpreting, but short memory is severely limited in size, it can only hold approximately seven plus or minus two information units.

Obviously, we cannot solely depend on our brain and memory while interpreting. Instead we should make good use 
of notes to help enlarge each unit and help record information as much and accurate as possible. From the above analysis, it can be concluded that recording stage in interpreting process must be fulfilled with the combination of effective mental memorization and necessary notes.

\section{The Encoding Procedure}

This procedure includes the activation of the target language (TL) elements found in the long-term memory, as well as syntactic and semantic word processing and word-string processing according to the TL syntactic and semantic information stored in long-term memory. The result is a paraphrase in TL of the source language message.

As long as the interpreter successfully accomplishes the first three procedures, factors that take effect on this procedure include the interpreter's second language proficiency, his master of various kinds of translation skills, and the intended receptors.

\section{E. The Expressing Procedure}

Expression is the terminal procedure in the process of interpretation. Although sound and effective expression can only be realized on the basis of successful fulfillment of the first four procedures, expression itself directly influences the final output and result, thus of great significance.

It is without doubt that the preceding procedures are the decisive factors for the success of the last procedure. However, there are still some points that the interpreter must pay attention in terms of the way of expressing. The interpreter's voice should be loud enough for everybody to hear, and it should be smooth and even, and the pitch should be appropriate. The interpreter's pronunciation of words should at least be good and constant, if not standard British or American English.

\section{BASIC INTERPRETING SKILLS}

\section{A. Listening Skills}

\section{Anticipation}

Anticipation is an important means that helps the interpreter to relieve the on-line memory load so that the processing capacity can be preserved for other efforts. From the aspect of language knowledge, anticipation can be generated from three levels, namely, the grammatical, syntactic and contextual levels.

At the grammatical level, anticipation can be activated by taking care of some signals offering clues for predicting what may come next to follow up such signals. Generally speaking, the signals will lead to set phrases and expressions. At the syntactic level, within a sentence, the meaning can sometimes be anticipated thanks to the presence of conjunctions which usually imply certain logic relationship between different parts of the sentences, such as although, therefore, etc. At the contextual level, the anticipation will be further expanded into a combination of several sentences or several paragraphs, which can be made based on the vocabulary, grammar rules, fixed phrases and idioms and the logical relations within the context.

The most important point is that all the anticipations are based on the topic-specific knowledge because all the meaning of the language comes from the combination of grammatical meaning and context meaning.

2. Improve psychological preparation

Effective listening is very important for comprehending the discourse. However, it is quite possible that the interpreters will get nervous when they are listening to the speaker's speech. As a matter of fact, getting nervous is inevitable for anyone who is going to interpret. While listening to the speaker, they should have a clear understanding of their identity and try to compose themselves as soon as possible before doing the interpreting job. The interpreters should set up confidence and enhance spirit to overcome difficulties.

The interpreters should learn to listen effectively. To listen effectively needs attention and concentration. Concentrating properly does not mean adopting a certain posture, frowning and straining with clenched fist, and it means to focus on the speaker's speeches.

When interpreters hear an idea that is not immediately clear to them, or miss an idea in the heat of the moment, the interpreters should keep calm and cautiously. At this time, the interpreters learn to continually fill in gaps as they listen, refer back on what they have already understood and understand difficult and unclear points by listening effectively to what is said.

\section{Use redundancy in listening training}

Interpreting work requires concentrated or discriminative listening, also known as active listening, which means avoiding all distractions and learning to be alert. The redundancy present in speech is a great advantage for the interpreters. In other words, they should learn to distinguish useful information from redundant information, and only concentrate their attention on the essential information.

Besides following the speaker's line of thought and catching the main idea, interpreters should also try to detect his/her attitude, mood and tone, recognizing special stylistic characteristics and rhetorical strategies used by him. These can provide non-linguistic information for the interpreter, thus forming the proper context for his speech, which might provide some hints for the interpreter in case he fails to catch one word or two during listening.

4. Use the expert or booth mate's help 
In consecutive interpreting, when an interpreter runs into comprehension problems, he may run to experts on site for help. Although the credibility of the interpreter may be reduced, this tactic can save the interpreter from misunderstanding and the serious problems in reconstruction.

In simultaneous interpreting, there are theoretically at least two interpreters in the booth at all times. One is active, while the other is passive. The passive colleague, who can devote full attention to listening, has a better chance of understanding difficult speech segment than the active interpreter, who has to share his attention into listening comprehension, short-term memory and reconstruction. Moreover, the passive interpreter has enough time to consult a glossary of other documents, and then give the information to the active interpreter, in writing or by murmuring.

5. Consult document during interpreting

An interpreter can also look for solution in documents, especially when there is no help from experts present or from the passive colleague available.

This tactic may be time-consuming and requires much processing capacity, but finding an important word in a document that had been read and marked before the conference can be very fast.

\section{B. Decoding Skills}

1. Cultivate familiarity with English pronunciations and dialects

As interpretation must be done orally, all the contents of interpretation soon fade away, interpreters should understand all the things the moment speeches come out. And owing to different geographic situation, cultural background and educational standard, people's pronunciation, intonation, wording and way of talking differ from each other. It is important to know the rule of pronunciation of source language and the feature of oral English. And sometimes interpreters have to work for non-native speakers, for example, Japanese, African, etc. Their pronunciations are generally hard to understand with the influence of their mother tongue.

Familiarity with different accents and dialects which is one of the symbols of senior interpreters can give some clues for interpreters during the course of interpretation so that they can fulfill interpreting tasks better. As non-native English speakers are trained in different places and organizations which have different pronunciation standards, and moreover, speeches made in international conferences often embodied features of Arabic, Nigerian and Spain English. Therefore, interpreters have to get themselves familiar with the diversity of English pronunciation and dialect.

2. Master a well-knit vocabulary system

Here, vocabulary includes not only ordinary words that can be found in dictionary, but also newly adopted words, abbreviations, slang and jargons. Nowadays, abbreviations are popular and common in the oral styles like oral communication and conference interpretations. The wide use of abbreviations had posed high requirements on interpreters. Except for the existence of abbreviations, English slang and jargons are hard nuts to crack to understand an English speech. Slang, an informal language, is often used in some informal situations, but they do appear in some non-official meetings and conferences.

Almost every interpreting assignment will challenge the interpreter with a complete new glossary of a certain field or discipline. Although understanding of special terms will not guarantee a successful interpretation, failure to do so will undoubtedly put an interpreter into a dilemma. As an interpreter is a generalist, to master a well-knit vocabulary is only the first step of accumulating background information.

3. Smooth shift of sentence structures between source language and target language

Theoretically, interpreters tend to have good command of languages and they know most syntax rules and sentence structures, but practically, "language specificity" often disturbs their thinking. Take English and Chinese as examples, attributives are pre-posed in Chinese, this is what linguists called "left-branching", while in English attributive clauses are often post-posed and "right-branching".

This difference may cause problems in interpretation. For instance, “姑苏号称水乡泽国，其水巷千姿百态，高低宽 窄错落有致, 尺度宜人富有韵律, 留恋岸边, 移舟水面, 宁静幽雅, 诗意盎然。”We have ample time in written translation and it can be put into English with a post-posed attributive clause. However, time is limited to form a sentence with well-knit structure, so interpreters should compromise to remain major information while give up its form.

4. Form logical analysis

Logical analysis can help facilitate comprehension, so it is necessary and important to train interpreter a logical analyzer. There are several ways to form logical analysis in interpretation. The first one is cognitive analyzing, in which interpreters relate the speaker' speech to their own knowing and understanding. The second one is analyzing according to position, in which interpreters make analysis based on their positions.

Each interpreter has his own views and judgment on certain things and remarks. These views and judgment can be helpful to understand the involving issues more deeply and accurately and easier to remember. But what worth mentioning is that no matter what attitude the interpreter has, his views cannot be revealed in the interpretation, for interpreters must interpret all the things in an objective, neutral manner, otherwise misinterpretation will occur.

5. Gradual Accumulation of ELK

Interpreting is a communication process that demands the interpreter have not only linguistic knowledge-phonology, lexis, syntax, semantics and texts which enables him to receive aural messages, but also extra-linguistic or encyclopedic 
knowledge (ELK) to help pave the way to efficient oral communication.

Extra-linguistic knowledge can be classified into three subcategories: knowledge of specific subject matter, knowledge of different interpreting situations and knowledge of cultural background. Subject knowledge refers to the basic knowledge for a particular field under discussion. Situational knowledge is also known as contextual knowledge which includes the background of speakers and audience, general information about the situation and some ideas of the working environment.

However, unlike linguistic knowledge, extra-linguistic language is much more subjective and dynamic. It can be pre-existing, for it is the knowledge which the interpreter has before he accepts a certain assignment and it can also be the knowledge which the interpreter takes from the context and the communicative situation. A good interpreter should be imbued with the spirit of the people who speak the language he is working with, familiar with their tradition, and closely acquainted with those literary works which have influenced the language. He should also appreciate their sense of humor, know the outstanding names of their history, sense the nuances of their styles as well as other euphemisms that may have lost their original meanings in part or in whole. To some extent, the interpreter could be regarded as a cultural bridge between people who are unfamiliar with each other's language and culture.

\section{Recording Skills}

In this procedure, the interpreter's memory plays a crucial role, and is a decisive factor in whether this procedure is successful or not or at least in how much the interpreter can get from the source's speech. A supplementary way of securing this procedure is note-taking, which seems to be more important in consecutive interpretation.

1. Visualizing memorization

Visualizing memorization is to visualize what the speaker is saying, for example, to form a picture or certain scene to accentuate memory. Such pictures and scenes are called situational models which are built on the basis of special or consequential relationship of contexts and thus acquire the longest time to be remembered.

Visualizing memorization is to visualize what the speaker is saying, for example, to form a picture or certain scene to accentuate memory. Such pictures and scenes are called situational models which are built on the basis of special or consequential relationship of contexts and thus acquire the longest time to be remembered.

The result of experiments showed that the capacity of memory of viewed pictures and image is much larger than that of words and speech. If interpreters can store the information of source speech as a single or a series of situational models rather than just words and sentences, they can memorize relatively more information with fewer symbols.

2. Outlining memorization

Interpretation is an oral communicative activity with strong purposes and be influenced by differed situations and discourses. Generally, each interpreting activity is focused on one or more clear topics and each speaker will keep consistence on the given topics. All these make outlining memorization possible.

Interpreters can make full use of the structure and main ideas of source language speech to outline the contents. In the outline or framework, what the interpreter memorized are the key points and the relationship among them. Later, the interpreter can use this outline or framework to activate relevant information in long term memory included. This skill is so useful that all the information can be completely generalized suitable for speeches to make arguments or introduction.

3. Reasoning memorization

Psychological and psycholinguistic experiments show that information is stored in form of abstract network. Reasoning memorization requires interpreters to make good use of the relationship among different information to combine bigger information units. This way of memorization is suitable for those boring speeches without good consistence.

4. Chunking

Chunking is a proven effective way to enlarge the short term store (STS) capacity in interpreting in that it allows interpreters to handle more information at one time. By recoiling the information held in STS with the activation of relevant information in long term store (LTS), new meaningful larger units of information that are familiar to interpreters are formed.

One way is to chunk the words into grammatical constituents such as nouns and verb phrases, thereby reducing the storage burden to perhaps two or three constituents. Chunking in interpreting is a process during which interpreters actively analyze, summarize and reorganize the messages of the speech. The external representation of chunking is to extract the key words from various layers of sense.

The key words can be either from the original speech or generated by interpreters to best summarize the intended meaning of a specific layer. In doing so, the memorization of a whole paragraph becomes the memorization of several key words. However, the collective use of key words cannot represent that full message intended in the speech since there do exist logical links between different layers of meaning as between different chunks.

Interpreters must, therefore, search for or generate the logical links during the chunking process besides extracting or generating the key words of messages. Key words are the pearls in a memory necklace while logical links are the thread that links the pearls. Together, they form the meaning structure of the full messages contained in speech. During the memorizing stage, interpreters mainly memorize the key words and logical links while in the recalling stage, they have to reactive them as the correspondent clues of the speech and then make out the sense of the speech they are going to 
interpret.

5. Note-taking skills

The success of interpretation is determined largely by interpreters' comprehensive recording capability. As there is a limit of human memory, so the recording capability includes "necessary note-taking" besides the mental recording of information in human mind. The notes taken for the purpose of interpretation are different from those written down by means of stenography. According to Li Kuiliu (1994), stenography is used to settle the contradiction between speech speed and recording speed, and it is the transformation between different ways of expression and involves only one language in its process while interpreters take notes to reveal original sense instead of original speech itself. Stenography needs great amount of attention and psychological concentration, however, interpreters must spare large portion of attention to realize the smooth comprehension and re-expression. What interpreters need to jot down in notes is words or symbols that can convey the important messages, clearly to recognize and can be told efficiently.

Generally speaking, interpreters should bring a pad of appropriate size with them which is easy to look through. And in order to make the notes convenient to be read out, the contents written down in each line should not be too much to avoid the occurrence of misunderstandings and misinterpretation. Space should be left after each phrase or sense group and diagonals must be added after each sentence, and when a comparative long paragraph is over, a line can be drawn to make the note clearer and help interpreters find the right place to start and finish interpreting units.

It is advisable that an interpreter should take notes vertically so as to break the sentences into sense units for easy digestion. Ladder structure pattern can vividly embody the logic order of the speaker. Taking notes in this way can help the interpreter follow the speaker's trace of thoughts. Thus he can perform the interpretation task more efficiently.

As far as the contents of the notes are concerned, first of all, what should be kept in the notes is the main idea of the original text. Secondly, it is words used to link or separate ideas like "and", "or", "but", etc. Thirdly, it is the point of view of the original text. Fourthly, the tense should be taken into consideration. And finally, it is contents such as numbers, dates, proper nouns, lists, etc.

It should be emphasized that what should be recorded in one's notes is not the actual words used in the original text, but its information. For example, numbers are very important in note-taking and they must be recorded accurately. However, what these numbers refer to is more important than these numbers.

Taking notes can help the interpreter remember the details and long sentences of a speech. However, in practicing note taking, many people will meet a dilemma, that is, sometimes taking notes will take away their focus, too. The only way to break through the bottleneck in note-taking training is to keep on practicing and learn to strike a balance. Good notes are always simple and easy, thus taking away the least focus possible.

Interpreters should not take notes for notes' sake. An interpreter may use either source language or target language or both in taking notes. He may choose the language that comes to his mind as the easiest or most appropriate one.

\section{Re-expression Skills}

\section{Timely adjustment}

Zhang Weiwei (1999) has asserted that interpreters can follow the principle of syntactic linearity during interpreting. It means that the interpreters follow the order of the source language, segmenting the input sentences into sense groups, concepts or information units and then naturally linking them together by some techniques so as to interpret the original meaning of the whole discourse. For example, in translating the sentence "I went to Holiday Inn for a seminar, at 10 o'clock yesterday.” According to the principle of syntactic linearity, we may interpret the sentence as“我去了假日酒店 参加一个研讨会, 在十点钟, 昨天。”This sentence sounds awkward. As far as the latter part of the sentence is concerned we had better make timely adjustment and add some words to it so as to make it sound more natural. The version“时间是在昨天上午10点”will be much better than the previous version.

By "timely adjustment" we mean interpreters should adjust the organization of language from time to time. Usually by making addition we can complement the meaning or tense or other minute information contained in the SL.

2. Adding information

Due to the difference in culture and habits, sometimes, interpreters need add some words or change the sentence structure to express the speaker's meaning. The following is an example.

The source speech: 特别是中下游河段, 水能集中, 交通方便, 工程量小, 地理位置好, 所以被国家列为十大 水电基地之一。

The target language: The middle and lower reaches of the river are particularly characterized by concentrated water energy, convenient transportation, small construction quantity as well as good geographical situation thus being listed as one of the ten largest hydropower basis in the country.

The interpretation adds one word "characterized", but the meaning becomes more clearly.

3. Reconstructing the segmental information in context

When the interpreter fails to hear or understand certain segmental information in the discourse, he can try to reconstruct it with the help of the context. His knowledge of language, his understanding of the subject and the situation he is in may also help him to reconstruct. If it is successful, the reconstruction can result in full recovery of the information.

However, since we need to allocate time and processing capacity on reconstruction, which may increase time 
pressure on the interpreter, reconstruction from the context can be used if needed, but it should not be considered as a highly priority tactic.

4. Reformulation

Due to the limitation of one's processing capacity, it is quite possible that the interpreter may miss some information when the information density is too high for him to cope with. On such occasions, the interpreter may reformulate the main idea in a general way. Though the accuracy of the discourse decreases, the audience can still get a whole picture of what the speaker has said. If the interpreter understands the meaning of the terms used in SL, but can hardly find the equivalent words in the TL, they can use paraphrasing or explaining method to get the meaning across.

5. Paraphrasing and explaining

For the so-called checkmates, it's impossible to find out an equal in TL in a short time, and the equal is not enough to make the audience understand the meaning of the source speech, in this case, paraphrasing and explaining are necessary.

In paraphrasing, the interpreters should obey the following rules:

a. They should not omit the facts given in the original text.

b. They should not change or alternate facts stated in the original text.

c. They should not slant attitude, stance, point of view, and tone that are stated explicitly in the original message.

Here are some paraphrasing tactics:

a. Changing the parts-of speech.

b. Changing the sentence structure.

c. Breaking up the long sentence into several short ones.

6. Reproducing the sound heard in the SL speech

When the interpreter comes across a proper noun or technical term which he can not recognize, he may reproduce the sound as heard. If the audience knows that term or proper noun, they may recognize it at once.

In conclusion, this section introduces some useful techniques that can be adopted in interpreting. Bearing these tactics in mind, the interpreter may tackle the problems in a more skillful way. However, these tactics cannot be overused, since they achieve successful reformulation at the sacrifice of overlooking some information.

\section{CONCLUSION}

This thesis probes interpreting skills from the perspective of interpreting process and proposes four main categories of skills to ensure the smooth fulfillment of the five procedures in interpreting process. It is hoped that this thesis will enable the language learners and those want-to-be interpreters to acquire a clearer understanding of interpreting skills and carry out relevant practices so as to make bigger progress.

\section{REFERENCES}

[1] Bao Gang. (1998). Introduction to Interpreting Studies. Beijing: Tourism Education Publishing House.

[2] Eugene A. Nida. (2001). Contexts in Translation. Shanghai: Shanghai Foreign Language Education Press.

[3] Guo Lanying. (2007). Studies on Interpretation and Interpreters Training. Beijing: Science Press.

[4] Li Kuiliu. (1994). Interpretation: Theory and Practice Language and Communication. Beijing: Foreign Language Teaching and Research Press.

[5] Liu Miqing. (1999). Theory of Translation. Beijing: China Translation \& Publishing Corporation.

[6] Li Yingying. (2005). Memory Mechanism and Memory Training in Interpreting. Ph.D. dissertation, Shanghai International Studies University.

[7] Yang Chunhui. (2004). The Information Processing in Oral Interpretation. Ph.D. dissertation, Central China Normal University.

[8] Zhang Weiwei. (1999). English-Chinese Simultaneous Interpretation. Beijing: China Translation \& Publishing Corporation.

Jing Ma was born in Taian, Shandong Province, China in 1979. She received her M.A. degree in linguistics from Shanghai Jiaotong University, China in 2005.

She is currently a lecturer in the School of Foreign Language, Qingdao University of Science and Technology, Qingdao, China. Her research interests include language testing, translation and interpretation. 